\title{
Wearable and Autonomous Computing for Future Smart Cities: Open Challenges
}

\author{
Domenico Balsamo, Geoff V. Merrett, Bahareh Zaghari, Yang Wei, \\ Sarvapali Ramchurn, Sebastian Stein, Alex S. Weddell, Steve Beeby \\ Department of Electronics and Computer Science \\ University of Southampton, United Kingdom, SO17 1BJ
}

\begin{abstract}
The promise of smart cities offers the potential to change the way we live, and refers to the integration of IoT systems for people-centred applications, together with the collection and processing of data, and associated decision making. Central to the realization of this are wearable and autonomous computing systems. There are considerable challenges that exist in this space that require research across different areas of electronics and computer science; it is this multidisciplinary consideration that is novel to this paper. We consider these challenges from different perspectives, involving research in devices, infrastructure and software. Specifically, the challenges considered are related to IoT systems and networking, autonomous computing, wearable sensors and electronics, and the coordination of collectives comprising human and software agents.

Index Terms-Smart cities, IoT, wearable computing, autonomous computing, human collectives.
\end{abstract}

\section{INTRODUCTION}

The vision of smart cities aims to integrate Internet of Things (IoT) technology to manage and improve urban development, the efficiency of services, and achieve a better quality of life [1]. This is illustrated in Fig. 1, where smart cities make use of an IoT-based intelligent environment, including peoplecentred services (e.g. parking and lighting, environmental monitoring). In this scenario, IoT is a networking paradigm that uses the internet to combine a large number of intelligent devices through standard communication protocols and provide information and services to end users [2]. As shown in Fig. 1, fundamental to this vision are networked wearable and autonomous devices, collections of large numbers of nodes organized into cooperative networks. Each device is typically equipped with sensors to detect physical phenomena such as light, heat, presence or pollutants; CPUs, DSPs to process data; an energy supply to provide power; memory for data storage; and a radio-frequency ( $\mathrm{RF})$ transceiver.

In this paper, we discuss the open challenges that need to be addressed in order to deliver on this vision. The novel approach taken by this paper is the consideration of the multidisciplinary research that is needed spanning devices, infrastructure and software. Specifically, we discuss the research challenges related to IoT sensor systems and networking with constrained and low-power systems (Section II), and then specifically focus on energy management of autonomous computing systems (Section III), wearable sensors and electronics (Section IV), and the coordination of collectives of humans and software agents (Section V).

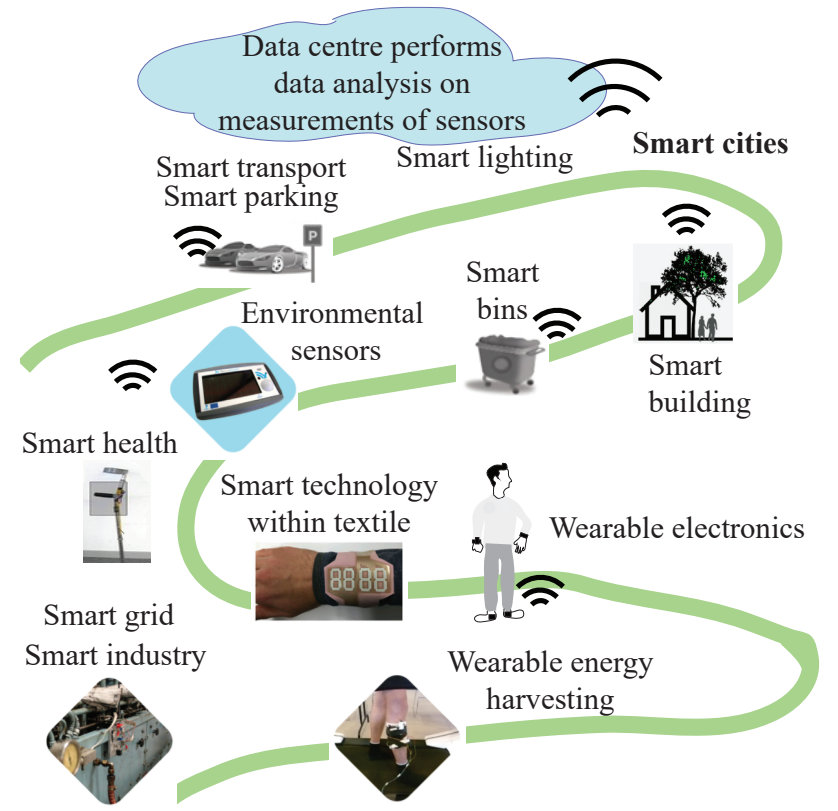

Fig. 1: A smart city intelligent environment using IoT to connect elements and provide services.

\section{IOT DEVICES FOR SMART CitIES}

Intelligent IoT devices are typically characterized by their inputs (including sensor and data acquisition), output display and data transmission, user interactivity and control, modularity and interoperability, integration into larger systems, and energy storage and efficiency. The future of smart cities depends on these aspects improving as a whole. While improvement in device design often requires compromise, existing technologies can be used in combination to produce superior systems.

As already underlined, rapid technological advances are driving widespread deployment of IoT devices in numerous application areas including environmental, health, transportation, smart buildings, and smart grids, etc (see Fig. 1). Some examples of intelligent devices are considered below with a particular focus on environmental sensing, with the aim of analysing challenges and opportunities.

Environmental sensors are required by smart cities, as they provide data input to the large-scale control systems. They measure a combination of parameters including temperature, 
pressure, wind, humidity, light, noise, and gas levels to inform pollution models. These pollution models can contribute to the design of transport networks. Environmental sensors also provide footfall information, and contribute data to weather prediction models. However, a compromise exists between the measuring capability of the device, its reporting frequency, and energy management (further in Section III), with the monetary cost of the device. A balance must be found between these factors to create a device that appeals to government bodies and companies.

Providing real-time information to humans that can be quickly understood is a key design driver for many devices. One example of this concept is a fire alarm system, where a loud tone is used to alert residents about an unexpected smoke level in a building. An enhancement of such a system could exist on a wearable device, which vibrates when the smoke level of a room exceeds a threshold. This wearable device could also synchronize with the building's wireless network on entry, to determine safe evacuation routes in the event of a fire, improving survival chances. Devices within homes can connect over an internal wireless network to disseminate information and instructions; for example a temperature control system, which manages heating in certain rooms to optimize the temperature. This technology operates on the basis that communication is free, because regular dissemination is required for temperature control. However, wireless communication across cities typically uses 4G networks, which are financially costly to use.

The challenges on providing wireless communication in a smart city are as follows:

Communication reliability: in an environment where millions of smart things are connected, challenges exist in achieving high-speed (and hence low-latency) while maintaining a reliable, efficient and secure communication.

Transmission infrastructure: many sensors are required to accurately map a parameter (such as traffic congestion) over a city, and these sensor nodes need to interact with each other wirelessly. These constrained nodes typically communicate to unconstrained gateway devices that relay on the information to the cloud. By relying on mobile telecommunications technology, expense is exacerbated by the number of devices and the amount of data they transfer. This is mitigated somewhat by advances in lower-range wireless communication protocols like ZigBee and LoRa, which have lower power requirements (only one percent of the power used in mobile telecommunications), but require investment in transmission infrastructure to operate [3], [4]. The compromise of reduced power (and hence range) using the limited range is that the devices have to operate cooperatively in order to enable multihop routing over longer distances when the packet size and the rate of communication is limited.

Energy availability versus communication requirements: a compromise is needed between the long-range, high-power, reliable, secure, and costly transmissions of $4 \mathrm{G}$ and $5 \mathrm{G}$ networks, and the short-range bespoke communications protocols [5]. This compromise depends on the energy and charging requirements of the device, and the structure of the device network. In order for smart cities to become viable investments for government bodies or companies, the cost of building, maintaining, and using long-range wireless infrastructure must be significantly reduced [1].

Smart cities rely on the characteristics of intelligent devices improving as technology evolves. The design of intelligent devices is becoming an increasingly interdisciplinary affair, with technologies combining in novel ways to satisfy the demands of the modern consumer. Once intelligent devices are able to communicate wirelessly at low cost over great distances, they can resolve the complex behavior that arises on the scale of cities, enabling the level of automation that smart cities promise to deliver.

\section{ENERGY MANAGEMENT}

Gathering information in a smart city typically requires systems that cannot always be connected to the power grid. A primary challenge is the energy management of devices as they typically have to last for many years, without intervention to charge or replace batteries [6]. Energy harvesting (EH) offers the potential for environmental systems to operate without batteries (autonomous devices), by generating electrical power from various sources including light, vibration, motion, or temperature differences.

However, systems with micro-power environmental energy harvesters have to accommodate the unpredictable nature of the sources. The power obtained from energy harvesters is dependent on the harvester, deployment location, and often on other factors such as weather, time of day, or machine activity. Kinetic or wind energy harvesters typically give an AC output relative to the frequency of vibration or rotation, while photovoltaic modules or thermoelectric generators typically give a more slowly-varying DC output. This problem is typically rectified through the addition of buffering energy storage to sustain computation and accommodate the mismatch between supply and consumption. This approach is known as energyneutral operation and attempts to balance long-term energy consumption against the harvested energy over a period of time (e.g. a day), such that the energy consumed equals the energy harvested [7]. However, due to the requirement for additional storage, these systems suffer from increased volume and cost.

In systems with tight volume constraints such as future flexible, wearable and implantable smart cities devices, this is something that is counter-productive. Such systems can, alternatively, include only a very small amount (or even zero) energy-storage. In this case, instead of the systems operation being dictated solely by the application, operation starts to become 'energy-driven', with execution and application functionality being highly intertwined with power and energy availability. The challenges of providing autonomous systems with minimal energy storage are as follows:

Power interruptions: systems are susceptible to frequent power interruptions and resets caused by the transient supply. A new paradigm, transient computing, enables computation to be sustained despite power outages [8]. This involves saving 
the system's state to Non-volatile Memory (NVM). Different transient computing approaches have been proposed to facilitate system state retention. However, these approaches are focused on saving only the state of the processing unit while they do not consider external peripherals such as transceivers and sensors, limiting the range of applicability. An open challenge is retaining the state of key external peripherals such as radio transceivers between power outages.

Energy-driven applications: for systems that operate directly from the energy harvesting source without using large additional energy storage, efficiently using the power provided by the source is fundamental. This requires the re-engineering of applications and systems, as the system's operation is not only driven by the application requirements (which are often temporally driven) but becomes dependent on power availability (which dictates operational times). For example, energydriven systems should consider the possibility of adjusting system's performance (and hence the power consumption) dynamically such that it matches the harvested power [9].

Networking with transiently-powered devices: There are two main challenges that need to be addressed when considering systems that rely on intermittently available energy:

1) Transient-powered systems don't control the time when they are on, while traditional battery-powered systems can rely on having all the nodes awake at the same time (or they can wait until all the nodes are on) to perform network communication. This is depicted in Fig. 2, where nodes in a transiently powered network cannot communicate, as neighbouring nodes are currently not powered. The challenge exists in designing asynchronous protocols which can accommodate this. Some approaches can be explored for these kind of systems such as opportunistic networking, which enables nodes to communicate with each other even if a route connecting them never exists [10], or wake-up radios [11].

2) Typical protocols for smart city systems require time to synchronize communication and introduce significant overheads over and above the data payload. This time can be larger than the time in which transient devices are on, and a challenge exists in drastically shortening it or splitting communication over multiple cycles.

\section{WEARABLE ElECTRONICS}

Electronic textile (e-textile) implementations offer the potential for unobtrusive wearable devices that are transparent to the wearer. However, there are many significant research challenges around achieving robust solutions. Wearable etextiles can incorporate sensing, actuating, communication and energy harvesting/storing functionality, as shown in Fig. 3.

The key criteria for wearable electronics are that they have to be robust, small, have low power/energy operation, and be comfortable to wearers [12]. E-textile based wearables require the electronics to be flexible, stretchable and washable. Another challenge is in achieving reliable connections between the components placed at different locations on the textile.

Fabrication: E-textiles can be realised by incorporating functional yarns into the textile or printing functional materials

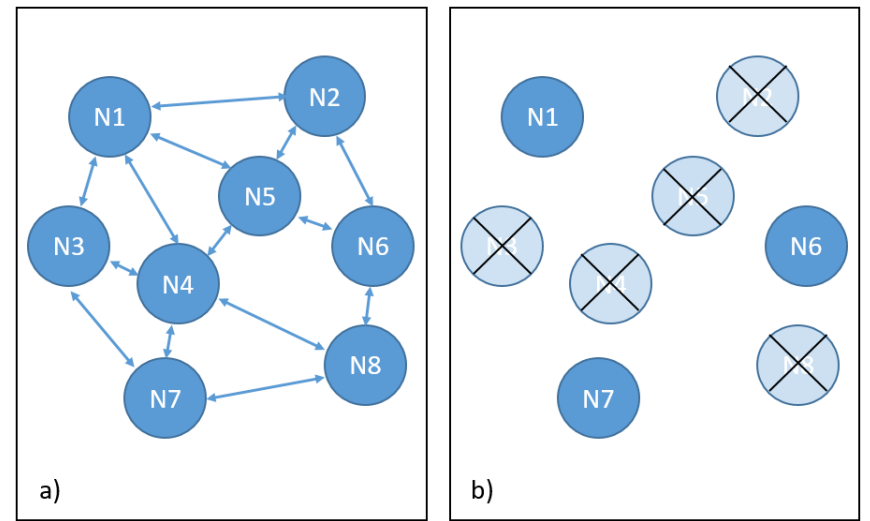

Fig. 2: Multi-hop synchronization with a) traditional connected network and b) transiently-powered 'sparse' network.

onto the textile surface. Functional yarns typically incorporate a single function and do not include full electronic circuits. A variety of functional materials can be printed, and entire circuits can be fabricated, on the surface of the textile. However, unlike other substrates, such as silicon and FR4 that have a smooth and flat surface, textiles have an uneven, porous and fibrous surface. This makes it extremely difficult to manufacture electronics directly onto textiles. An additional coating on top of the fabric can be used to planarise the surface and these can be laminated or printed to the textile [13], [14]. Electronic components and interconnects can be incorporated onto this surface. This approach, however, does alter the properties and feel of the textile and thus potentiality reduces the comfort to the wearer. Circuit reliability is also a concern under mechanical loads.

Materials must be flexible, and withstand repeated bending as the textiles flex. Any physical damage to the materials will impact the performance of entire device. In addition, the processing conditions of the materials and circuits must not damage the original properties of the textiles. High temperature and harsh environments (e.g. the etching process) must be avoided because the fabric properties may be degraded.

Interconnection: reliable interconnections between electronic subsystems such as sensors, circuits, antennas and batteries, are critical. Those devices may be positioned separately from each other around the body. The interconnection has to be flexible and not restrict the movement of the wearer. Interconnection joints where the flexible substrate meets rigid components are a particular challenge due to the concentration of stress at these locations.

Reliability: one of the key challenges of e-textiles is reliability during daily use and washing. In addition to the flexibility described previously, the materials used for fabrication must not degrade over time during the lifetime of the wearable electronics. Washing is a very harsh environment for wearable e-textiles causing mechanical strain, abrasion as well as exposure to water and detergents. Although the research into waterproof material as the encapsulation has been reported [13], washability of wearable electronics is still a key 
challenge to resolve.

In the following, we consider typical open challenges in RFID,air-quality sensing and energy harvesting in wearable applications.

1) Wearable RFID: Radio Frequency Identification Devices (RFID) generally consist of transponders associated to each object to be detected, a reader that sends/receives information from the transponders, and a database that gathers the information to be processed [15]. A RFID transponder consists of a chip and associated antenna of which the impedance must match with the chip impedance at a particular frequency to maximize the power transmission through backscattering [16]. The frequency for which RFID operates varies depending on the detection range and power required for particular applications and geographic locations. The key challenges of integrating RFID technology within textiles are maximizing the detection range, minimizing overall size, improving transmission directivity and optimizing/developing fabrication and implementation techniques.

Antenna performance: wearable RFIDs have to be flexible, lightweight, robust, and easily integrated within the textile in a non-obtrusive manner. The properties of the antenna materials and the textile influence the behavior of the antenna such as the bandwidth and efficiency.

Body movement: the movement of the body can deform the geometry of the antenna and affect its performance [16]. For example, when the textile is bent and deformed, the electromagnetic properties of the antenna are also changed, thus influencing the overall RFID performance.

2) Air Quality Monitoring: People spend more than half of their time in the office [17]. Wearable air quality sensors in an office environment allow particular gas concentrations to be dynamically monitored and mapped in real time. The data can be combined with intelligent management systems to provide distributed control of the environment within the building to improve building services (e.g. heating and ventilation).

Air quality sensors are mainly based on the principle of electrical variation between different materials under the application of a particular air pollutant(s) [18]. However, one of the key issues is that a heater is needed in order to provide an optimised operating temperature for which the sensing material starts to function. The temperature required can be up to $450 \mathrm{C}$ [19] which is difficult to be integrated within textiles and consumes a significant amount of energy.In addition, the recovery time of air quality sensors is sometimes around 100 times longer than its response time (approximately 1s), and therefore pollution variation is difficult to be updated in real time [20].

3) Wearable Energy Harvesting: As mentioned in Section III, harvesting energy from the wearer or the surrounding environment allows continuous powering to wearable electronics without the need for batteries. Energy can be harvested from the kinetic motion of the wearer, on-body temperature differences and solar energy around the wearer.

Energy conversion: the key challenges of wearable energy harvesting are the minimal generated power and continual-

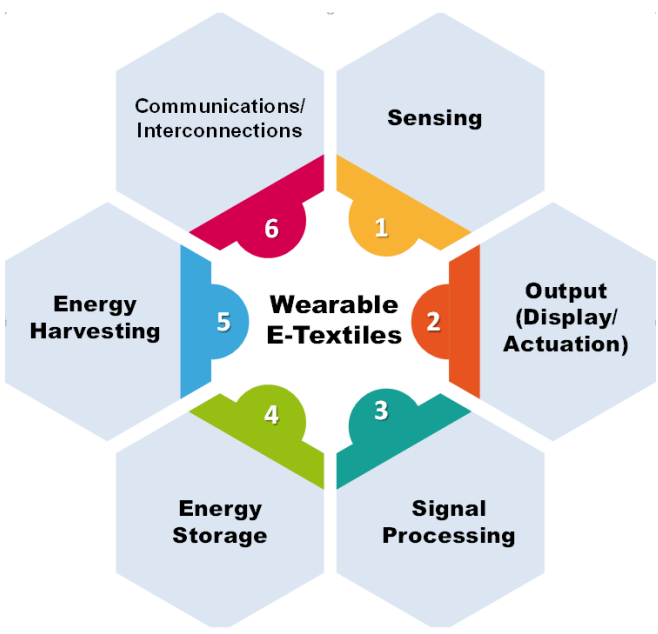

Fig. 3: Wearable e-textile system.

ity. For harvesting through kinetic motion, piezoelectricity is commonly used as the transduction mechanism to convert mechanical vibration to electricity. The conversion efficiency strongly depends on the material properties, harvester geometry, installation location on human body, magnitude of body vibration, and the associated circuity [21]. Piezoelectric materials can be deposited onto textiles to convert energy from the textile deforming but energy levels are low at around $100 \mu J$ per action [22]. For thermoelectric harvesters, the challenge is to maximize the temperature difference across the device, but this is not easily achieved on the human body [23]. Low-temperature flexible thermoelectric materials that could be combined with textiles are being investigated, but these are not yet practical solutions [24]. For solar energy harvesters, the harvested energy strongly depends on the solar radiation, efficiency of photovoltaic element, the location of the harvester as well as the location of the wearer (e.g. indoor or outdoor) [17]. Integrating the functionality into the textile is not straightforward, and at present the only possible approach is to attach plastic cells to the textile [25].

\section{SMART SOFTWARE}

A key step for realizing our vision of future smart cities is the design of smart software. This software will assist in the efficient collection of data from sensors and wearable devices, and it will help users make sense of the vast amount of information that will be at our disposal. We envisage that smart software will be ubiquitous across the smart city infrastructure, running on low-powered sensors and devices, advising and engaging citizens via smartphone apps, aggregating and filtering streams of data and assisting city planners in achieving situational awareness and making the right decisions.

Yet, in designing such smart software, several open research challenges will need be addressed:

Dealing with a deluge of data: due to bandwidth, processing and battery constraints, techniques are needed that can detect key signals and filter out unwanted noise or redundant information. An important aspect of this is how to deal 
with the high dynamism and uncertainty of the data. This includes coping with delayed or incomplete data, and data being gathered at different resolutions (spatial and temporal) from multiple sensors.

Incentivising participants: as citizens will contribute sensor data via wearable devices, appropriate incentives may need to be offered [26]. These could also be used to deal with malicious behaviour, where users intentionally provide false data, or to coordinate the sensing actions of participants, e.g., to direct them to areas of high interest or to avoid taking redundant measurements [27].

Achieving situational awareness and improving decisionmaking: in order to effectively exploit the vast information that will be available, smart software needs to be able to extract, summarise and then clearly present key information to decision-makers, so that they can take appropriate actions. We envisage that this can be achieved through socio-technical partnerships called human-agent collectives, where software agents and human decision-makers work together to identify the best course of action in a given situation [28]. In many cases, such actions can be partially or completely automated via the agents (e.g., diverting traffic or issuing incentives for using public transport when pollution levels reach critical levels), but in other cases, control may need to be given to human actors (e.g. to deal with complex emergencies or planning long-term infrastructure investments).

We envisage that these challenges can be addressed through the development of new machine learning techniques for dealing with large amounts of data on low-powered devices (data deluge); through new incentive engineering techniques that combine work from human computation and behavioural economics (participant insentivisation) and through new algorithms and interaction mechanisms for human-agent collectives in the presence of vast and uncertain data (situational awareness/decision making).

\section{CONCLUSION}

In this paper, we have discussed the different challenges that need to be addressed to deliver on the vision for wearable and autonomous devices for IoT-based smart cities. This involves a multidisciplinary approach, which requires research on devices, infrastructure and software. Open research challenges were specifically focused on IoT devices and systems, energy management and wireless communication. We explored autonomous computing systems and wearable devices, and the challenges related to smart software for the coordination of human collectives and software agents.

\section{ACKNOWLEDGMENT}

This work was supported by the UK Engineering and Physical Sciences Research Council (EPSRC) under Grant EP/P010164/1.

\section{REFERENCES}

[1] H. Dawid et al. "Management science in the era of smart consumer products: challenges and research perspectives," Central European J. of Operations Research, 203-230, 2017.
[2] L. Mainetti et al. "Evolution of wireless sensor networks towards the internet of things: A survey," in IEEE Int. Conf. on Software, Telecommun. and Comput. Networks, 2011.

[3] P. Yi et al. "Developing ZigBee deployment guideline under WiFi interference for smart grid applications," IEEE Trans. on smart grid, 110$120,2011$.

[4] A. Augustin et al. "A study of LoRa: Long range and low power networks for the internet of things," Sensors, 2016.

[5] M. Dohler et al. "Machine-to-machine in smart grids and smart cities technologies, standards, and applications," Tutorial Globecom, 2012.

[6] J. W. Matiko et al. "Review of the application of energy harvesting in buildings," Measurement Science and Technology, 25(1), 2013.

[7] V. Sharma et al. "Optimal energy management policies for energy harvesting sensor nodes," IEEE Trans. on Wireless Commun., vol. 9, no. 4, pp. 13261336, April 2010.

[8] D. Balsamo et al. "Hibernus: Sustaining computation during intermittent supply for energy-harvesting systems," IEEE Embedded Syst. Lett., 2015.

[9] D. Balsamo et al. "Graceful Performance Modulation for Power-Neutral Transient Computing Systems," in IEEE Trans. Comput.-Aided Des. Integr. Circuits Syst., vol. 35, no. 5, pp. 738-749, May 2016.

[10] L. Pelusi et al. "Opportunistic networking: data forwarding in disconnected mobile ad hoc networks," in IEEE Commun. Mag., vol. 44, no. 11, pp. 134-141, November 2006.

[11] M. Magno et al. "Design, Implementation, and Performance Evaluation of a Flexible Low-Latency Nanowatt Wake-Up Radio Receiver," in IEEE Trans. Ind. Informat., vol. 12, no. 2, pp. 633-644, April 2016.

[12] X. Tao et al. "Wearable Electronics and Photonics," Cambridge: Woodhead Publishing, 2005.

[13] K. Yang et al. "Waterproof and durable screen printed silver conductive tracks on textiles," Textile Research J., pp. 2023-2031, 2013.

[14] DuPont Stretchable Electronic inks, Available at: http://www.dupont.com/products-and-services/electronic-electricalmaterials/printed-electronics/products/stetchable-inks-for-wearableelectronics.html [Accessed: 11-08-17].

[15] A. Ghiotto et al. "Chip and antenna impedance measurement for the design of passive UHF RFID tag," in Proc. of the 40th European Microwave Conf., Paris, 2010.

[16] G. Marrocco "The art of UHF RFID antenna design: impedancematching and size-reduction techniques," IEEE Antennas \& Propagation Mag., vol. 50, pp. 66-79, 2008.

[17] M. Stoppa and A. Chiolerio "Wearable electronics and smart textiles: A critical review," Sensors, vol. 14, pp. 11957-92, 2014.

[18] J. Samet et al. "Health effects and sources of indoor air pollution. Part II," The American Review of Respiratory Disease, vol. 137, pp. 221-42, 1988.

[19] X. Liu et al. "A survey on gas sensing technology," Sensors, vol. 12, pp. 9635-65, 2012.

[20] S. Majumder et al. "Liquid petroleum gas sensor based on $\mathrm{SnO} / \mathrm{Pd}$ composite films deposited on $\mathrm{Si} / \mathrm{SiO} 2$ substrates," Vacuum, vol. 81, pp. 985-96, 2007.

[21] S. Beeby et al. "Energy harvesting vibration sources for microsystems applications," Measurement Science and Technology, vol. 17, pp. R17595, 2006.

[22] A Almusallam et al. "Flexible piezoelectric nano-composite films for kinetic energy harvesting from textiles," Nano Energy, 33, pp. 146-156. 2017.

[23] V. Leonov "Thermoelectric energy harvesting of human body heat for wearable sensors," IEEE Sensors J., vol. 13, pp. 2284-91, 2013.

[24] Z Cao et al. "Screen printable flexible BiTe-SbTe based composite thermoelectric materials on textiles for wearable applications," IEEE Trans. on Electron. Devices, 63, (10), pp. 4024-4030, 2016.

[25] R Mather and John Wilson, "Fabrication of Photovoltaic Textiles, Coatings, 7(63), 2017.

[26] H. Gao et al. "A Survey of Incentive Mechanisms for Participatory Sensing," IEEE Commun. Surveys \& Tutorials, 17(2). pp. 918-943, 2015.

[27] A. Zenonos et al. "A Trust-Based Coordination System for Participatory Sensing Applications," Proc. of the 5th AAAI Conf. on Human Computation and Crowdsourcing, 2017. [In press]

[28] N. R. Jennings et al. "Human-agent collectives," Commun. ACM 57(12), pp. 80-88, 2014. 